\title{
Model-Based Estimation With Boundary Side Information or Boundary Regularization
}

\author{
Ping-Chun Chiao, W. Leslie Rogers, Jeffrey A. Fessler, Neal H. Clinthorne, and Alfred O. Hero
}

\begin{abstract}
We have previously developed a model-based strategy for joint estimation of myocardial perfusion and boundaries using ECT (Emission Computed Tomography). We have also reported difficulties with boundary estimation in low contrast and low count rate situations. In this paper, we propose using boundary side information (obtainable from high resolution MRI and CT images) or boundary regularization to improve both perfusion and boundary estimation in these situations. To fuse boundary side information into the emission measurements, we formulate a joint log-likelihood function to include auxiliary boundary measurements as well as ECT projection measurements. In addition, we introduce registration parameters to align auxiliary boundary measurements with ECT measurements and jointly estimate these parameters with other parameters of interest from the composite measurements. In simulated PET O-15 water myocardial perfusion studies using a simplified model, we show that the joint estimation improves perfusion estimation performance and gives boundary alignment accuracy of $<0.5 \mathrm{~mm}$ even at 0.2 million counts. We implement boundary regularization through formulating a penalized log-likelihood function. We also demonstrate in simulations that simultaneous regularization of the epicardial boundary and myocardial thickness gives comparable perfusion estimation accuracy with the use of boundary side information.
\end{abstract}

\section{INTRODUCTION}

$\mathbf{E}^{\mathrm{c}}$ CT (Emission Computed Tomography) quantification of cardiac physiological parameters such as myocardial perfusion benefits from accurate delineation of endocardial and epicardial boundaries and accurate estimation of radiotracer concentration in each region of interest (ROI). We have previously [1] developed a strategy for joint estimation of physiological parameters and myocardial boundaries. We have also discussed difficulties with myocardial boundary estimation in simulated low count rate situations. When the number of counts for the entire study is below 0.4 million, the estimation process becomes unstable. For instance, we have occasionally obtained angular myocardial thickness estimates near zero using a 16-node model at 0.2 million counts.

Our specific concern about low count rate situations is motivated by electrocardiogram (ECG) gating techniques [2], which can freeze heart motion and consequently improve the definition of myocardial boundaries and ROI concentrations in a beating heart. In a gated study, each cardiac cycle is timed

Manuscript received November 7, 1991; revised August 10, 1993. This work was supported in part by National Cancer Institute under Grant RO1 CA32846 and by Squibb Diagnostics, Princeton, NJ. The associate edito responsible for coordinating the review of this paper and recommending its publication was R. Leahy.

The authors are with the Division of Nuclear Medicine, University of Michigan, Ann Arbor, MI 48109 USA.

IEEE Log Number 9401112 and divided into $K$ time frames according to ECG signals. The multiple gated ECT data are simultaneously acquired and partitioned into these gated $K$ groups. Since the heart is relatively stationary within each time frame, $K$ sets of region parameters will adequately describe the dynamic boundaries. However, estimating boundaries in each group will be a challenging task because each group has roughly only $1 / K$ total counts.

The use of auxiliary high resolution images such as those from MRI (Magnetic Resonance Imaging) and CT (Computed Tomography) has been proposed to improve region delineation on PET brain images [3]-[6]. We expect that boundary side information can substantially improve joint estimation of physiological parameters as well as myocardial boundaries in cardiac studies. Immediate questions related to this side information are (1) how should this information be aligned with ECT measurements and (2) how should the information be used? Even though techniques for aligning auxiliary boundary measurements have been proposed [3][6], they require additional effort such as surface fitting [3], external localizing devices [4], and elastic matching using a digitized atlas [5], [6]. Though algorithms for fusing boundary side information into ECT image reconstruction have been proposed [7], [8], methods for optimal use of boundary side information in physiological parameter quantification have not been developed that account for the facts that (1) boundary side information is always imperfect in practice, and (2) the process of aligning this information will itself be subject to significant error.

Our problem with boundary parameter estimation in low count rate and low contrast situations has similar characteristics to the ill-posed inverse problem often encountered in edge detection [9], computer vision [10], image restoration [11], and image reconstruction [12]. Ill-posedness results from too many parameters, poor data sampling, and weak inherent information (low contrast and signal to noise ratios). A common technique for solving ill-posed problems is "regularization" which modifies the objective function so that obtainable solutions satisfy some desirable criteria such as smoothness.

To overcome difficulties in myocardial boundary estimation in low count rate and low contrast situations, in this paper we extend the model-based joint maximum likelihood estimator (MJMLE) [1] to include myocardial boundary side information and to impose smoothness constraints on boundary parameters. To fuse the boundary side information into the emission measurements, we formulate a joint log-likelihood function to include auxiliary boundary measurements as well as ECT 
projection measurements. In this manner, we incorporate not only auxiliary boundary estimates but also the statistical descriptions about these estimates. In addition, we introduce registration parameters to align the auxiliary measurements with the emission measurements and jointly estimate these parameters with other parameters of interest from the composite measurements. To impose the smoothness constraints, we implement myocardial boundary regularization through formulating a penalized log-likelihood function.

\section{BOUNDARY SIDE INFORMATION}

We use an object model that consists of two parts: a heart and the rest of body. We geometrically model the heart using a polygonal model [1] and parameterize myocardial boundaries by a set of endocardial radii and a set of angular thicknesses with respect to an origin chosen toward the center of the heart. By assuming the body contour is exactly known, we incorporate only auxiliary myocardial boundary measurements. Extension to incorporating auxiliary whole body boundary measurements is discussed in Section VII.

In general, alignment of ECT measurements with MRI or CT measurements involves translation, rotation, and scaling. In the following formulation, we consider only $2 \mathrm{D}$ translation. Methods for dealing with rotation and scaling are discussed in Section VII.

We assume that auxiliary epicardial and endocardial radius measurements concatenated in a vector $\tilde{\mathbf{R}}$ are independent of ECT measurements $\tilde{\mathbf{Y}}$. Thus, the joint log-likelihood of the two sets of measurements $\ln f_{\mathbf{Y R}}$ is the sum of the two individual log-likelihoods:

$$
\begin{aligned}
\ln f_{\mathbf{Y}, \mathbf{R}}(\tilde{\mathbf{Y}}, \tilde{\mathbf{R}} ; \mathbf{A}, \mathbf{S}, \mathbf{X})= & \ln f_{\mathbf{Y}}(\tilde{\mathbf{Y}} ; \mathbf{A}, \mathbf{S}, \mathbf{X}) \\
& +\ln f_{\mathbf{R}}\left(\tilde{\mathbf{R}} ; \mathbf{R}=\mathbf{G}^{-1} \cdot \mathbf{S}\right) .(1)
\end{aligned}
$$

In (1), the primary parameters $\mathbf{A}$ include compartmental parameters, blood pool concentrations, and background concentrations [1]. The registration parameter vector $\mathbf{X}$ consisting of coordinates $x_{1}$ and $x_{2}$ characterizes the transformation between a polar coordinate system for the auxiliary measurements and a Cartesian coordinate system for the ECT measurements (Fig. 1). To impose positivity constraints [1], we parameterize myocardial boundaries using endocardial radius and angular thickness parameters, which are concatenated in a vector $\mathbf{S}$. In addition, for convenience we characterize mean auxiliary boundary measurements using endocardial and epicardial radius parameters concatenated in $\mathbf{R}$. The relation $\mathbf{G}$ between $\mathbf{S}$ and $\mathbf{R}$ is described in Appendix $\mathbf{B}$. $\ln f_{\mathbf{Y}}$ and ln $f_{\mathbf{R}}$ and are the log-likelihoods of observing $\tilde{\mathbf{Y}}$ and $\tilde{\mathbf{R}}$, respectively. MJMLE proposed in [1] can be directly applied to jointly compute estimates for $\mathbf{A}, \mathbf{S}$, and $\mathbf{X}$ through maximizing $\ln f_{\mathbf{Y}, \mathbf{R}}(\tilde{\mathbf{Y}}, \tilde{\mathbf{R}} ; \mathbf{A}, \mathbf{S}, \mathbf{X})$ with respect to the three sets of parameters.

One may imagine $\hat{\mathbf{R}}$ as a nonrigid frame (Fig. 1), which will be matched and fitted to the object in the process of joint estimation. The rigidity of the frame depends on the accuracy of the auxiliary measurements: the more accurate the measurements are, the more rigid the frame is.

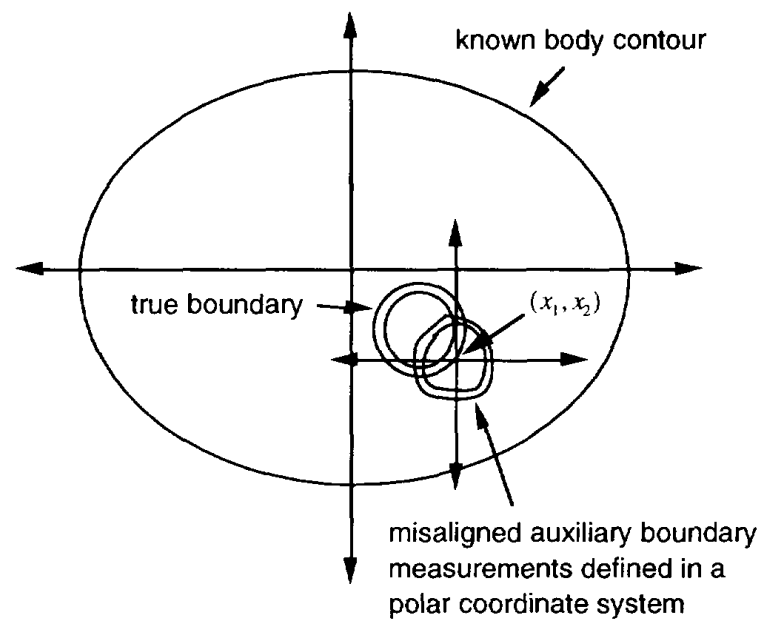

Fig. 1. Coordinate systems for ECT and auxiliary boundary measurements.

\section{BOUNDARY REGULARIZATION}

For the purpose of this paper, we will not discuss the theoretical issue of regularization. Rather, we demonstrate the power of this method in our formulation and simulation results. Readers may refer to [13] for theories and to [9]-[12] and references therein for instructive application examples.

Regularization provides a convenient way to stabilize parameter estimation. It does so through a regularization functional that controls the smoothness of obtainable solutions. We use this functional to regularize boundary parameter estimates by penalizing the ECT measurement $\log$-likelihood $\ln f_{\mathbf{Y}}$. Thus, the objective function in this case is a penalized loglikelihood $g$

$$
g(\tilde{\mathbf{Y}} ; \mathbf{A}, \mathbf{S}, \alpha)=\ln f_{\mathbf{Y}}(\tilde{\mathbf{Y}} ; \mathbf{A}, \mathbf{S})-\alpha \cdot \Delta \mathbf{R}^{T} \cdot \mathbf{W} \cdot \Delta \mathbf{R}
$$

in which the quadratic functional $\Delta \mathbf{R}^{T} \cdot \mathbf{W} \cdot \Delta \mathbf{R}$ measures the roughness of radius parameters $R, \Delta$ denotes finite difference (see Appendix B), $\mathbf{W}$ is a prescribed nonnegative definite matrix weighting the elements of $\Delta \mathbf{R}$ (i.e., specifying where in $\mathbf{R}$ to smooth more or less), and $\alpha>0$ is a regularization parameter. Maximization of (2) presents two conflicting goals: maximization of $\ln f_{\mathbf{Y}}$ and minimization of $\alpha \cdot \Delta \mathbf{R}^{T} \cdot \mathbf{W} \cdot \Delta \mathbf{R}$. The regularization parameter $\alpha$ controls the trade-off between the two goals [14], [15]. If $\alpha$ is too large, the obtainable solutions are smooth but may not agree with the data well, which may cause a systematic error (bias). On the other hand, if $\alpha$ is too small, regularization may fail to stabilize the estimation process.

\section{Simulation}

We have simulated low contrast PET O-15 water myocardial perfusion studies at 0.2 million counts to evaluate the performance of joint estimation with boundary side information or boundary regularization. MJMLE, which implements constrained Fisher scoring and Marquardt's interpolation method [1], was used to maximize the objective functions derived in 
TABLE I

MODEl CONFIGURATIONS, DATA SAMPLING SCHEMES, AND SPECIFICATION OF BOUNDARY SIDE INFORMATION

- Object model for joint estimation with boundary side information

- 2 concentric polygons (each has 16 nodes connected by straight lines) and 1 large ellipse, forming 6 uniform regions: blood pool, 4 myocardial regions, and the rest of body.

- The 4 myocardium regions are each independently parameterized by a set of compartmental parameters.

- Radius of endocardial nodes $r_{a}=4 \mathrm{~cm}$.

- Radius of epicardial nodes $r_{b}=5 \mathrm{~cm}$.

- Elliptical body outline dimension $=14 \mathrm{~cm} \times 17 \mathrm{~cm}$.

- 10\% vascular space in myocardium.

- Nominal plasma concentrations were generated from analytical functions [19].

- Concentration of the rest of body $=1 / 10$ plasma concentration.

- No attenuation, random coincidence, deadtime, and scatter.

- Object model for joint estimation with boundary regularization

- All the descriptions are the same as the model above except elliptical endocardial dimension of $3 \mathrm{~cm} \times 4 \mathrm{~cm}$ and elliptical epicardial dimension of $4 \mathrm{~cm} \times 5 \mathrm{~cm}$.

- Compartmental modeling

- Kety's 2-compartment model.

- Tissue-blood partition coefficient $p=0.9$ (ml/gm).

- $k \mathrm{l}=1.2(\mathrm{ml} / \mathrm{gm} / \mathrm{min})$ for each myocardial ROI.

- $k 2=k 1 / p=1.33(/ \mathrm{min})$ for each myocardial ROI.

- ECT system model and data sampling schemes

- 64 detectors, parallel ray collimation.

- System resolution $=10 \mathrm{~mm}$ (FWHM).

- Total counts $=0.2$ million, unless explicitly specified with a different value.

- 10 time intervals: $6 \times 10 \mathrm{sec}, 3 \times 1 \mathrm{~min}$, and $1 \times 4 \mathrm{~min}$; total time $=8 \mathrm{~min}$.

- 8 angular projections per $180^{\circ}$ in every simulation.

- 32 angular projections per $180^{\circ}$ for generating filtered-back projection images.

- Boundary side information (auxiliary radius measurements)

- Independent of ECT measurements.

- Errors characterized by a multidimensional Gaussian distribution with zero-mean and a covariance structure (see Appendix A) with $\sigma=1 \mathrm{~mm}$ and $\rho=0.3$.

(1) and (2). We also address the following questions concerning the alignment of myocardial boundary side information:

1) How well does joint estimation of primary parameters $\mathbf{A}$, boundary parameters $\mathbf{S}$, and registration parameters $\mathrm{X}$ compared to perfect alignment of imperfect boundary side information?

2) How does it affect myocardial perfusion estimation to simply delineate ROIs using auxiliary myocardial boundary measurements even with $1 \mathrm{~mm}$ registration accuracy?

For the simulations related to these questions, we define three methods:
Method 1- Estimation of primary parameters A (ROIs are fixed [16], [17] and are specified from imperfect boundary side information with registration root-mean-square error of $1 \mathrm{~mm}$ ).

Method 2- Joint estimation of primary parameters $\mathbf{A}$ and myocardial boundary parameters $\mathbf{S}$ with imperfect boundary side information and perfect registration.

Method 3- (emphasized in this paper)-Joint estimation of primary parameters $\mathbf{A}$, boundary parameters $\mathbf{S}$, and registration parameters $\mathbf{X}$ with imperfect boundary side information. 


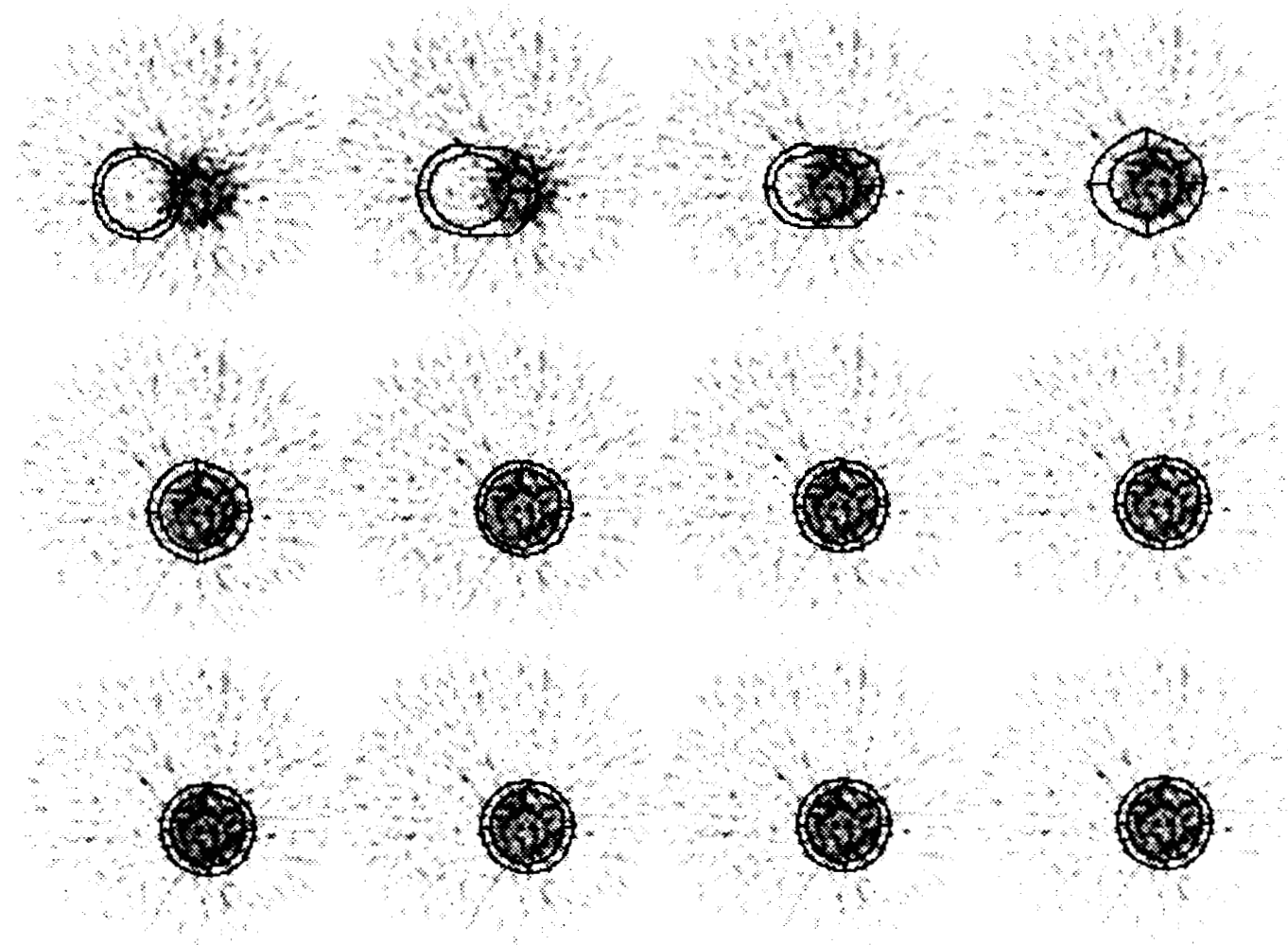

Fig. 2. The iteration process using Method 3 (from left to right, top to bottom), which starts with misaligned auxiliary measurements (shifted to the left by $6 \mathrm{~cm}$ ) and ends in well-matched boundary estimates. The final boundary estimates differ from the initial estimates because the final estimates extract information from the composite auxiliary and ECT measurements.

For the boundary regularization method, we investigate the following issues:

1) Is perfusion estimation sensitive to the selection of the regularization parameter?

2) What is the best value of $\alpha$ for boundary estimation?

3) Is boundary regularization as useful as boundary side information?

We assume that ECT measurements $\tilde{\mathbf{Y}}$ have Poisson distributions and that auxiliary boundary measurements $\tilde{\mathbf{R}}$ have a multidimensional Gaussian distribution with mean $\mathbf{R}$ and a Markov-type covariance matrix (see Appendix A). We also assume that auxiliary boundary measurements are either drawn by human operators or determined by edge detectors that ensure boundary continuity. In addition, we assume that the resulting endocardial and epicardial curves will be converted to radius measurements in a polar coordinate system with prespecified angular separation.

We choose to simultaneously regularize epicardial radii and angular thicknesses for the following reasons: (1) the epicardial boundary is smoother than the endocardial boundary; (2) The contrast at the epicardial boundary is usually higher than that at the endocardial boundary in perfusion studies, meaning that the epicardial boundary is less vulnerable to oversmoothing; (3) the left ventricular myocardium has approximately uniform thickness. This regularizer and its
Hessian, which is required in implementing MJMLE, are described in Appendix B.

Table I summarizes model configurations, data sampling schemes, and specification of boundary side information. The polygonal heart model has been described in detail in [1], [18]. In all simulations, the myocardium was subdivided into 4 ROIs, each of which was individually parameterized by $k: 1$ (perfusion) and $k 2$ based on Kety's 2-compartment model. The boundaries between the myocardial ROIs were prespecified with equiangular separation and were fixed during estimation. For simulations related to boundary regularization, we used an elliptical heart model to facilitate the demonstration of biases introduced by improper selection of the regularization parameter $\alpha$. The number of 0.2 million counts was based on an $8 \mathrm{~min}$ PET $\mathrm{H}_{2}^{15} \mathrm{O}$ study that amounts to a total of $\sim 1.5$ million counts/slice for the entire study, and the number was estimated for 1 out of 8 groups of multiple gated measurements. The specification $(\sigma=1 \mathrm{~mm}$ and $\rho=0.3)$ of boundary side information was intended to characterize the inherent uncertainty of boundaries extracted from MRI or CT images and distance dependence correlations among radius measurements.

In all simulations, the initial estimates of perfusion, blood pool concentrations, and background concentrations were generated from independent Gaussian distributions with standard 
TABLE II

STATISTICS OF AND REGISTRATION PARAMETER ESTIMATES AND COMPARISON OF SIDE INFORMATION WITH REGULARIZATION (NUMBER OF REALIZATIONS $=180$ FOR EACH CASE, TOTAL COUNTS $=0.2$ MILLION)

\begin{tabular}{|c|c|c|c|}
\hline & bias & $\mathrm{SD}$ & RMS \\
\hline $\begin{array}{cc} & k 1 \text { of ROI } 1 \\
\text { Method 1 } & 2 \\
& 3 \\
& 4\end{array}$ & $\begin{array}{l}2.72 \pm 1.94(\%) \\
4.80 \pm 1.81 \\
3.41 \pm 1.94 \\
3.97 \pm 1.88\end{array}$ & $\begin{array}{l}26.04 \pm .69(\%) \\
24.32 \pm .64 \\
26.01 \pm .69 \\
25.22 \pm .67\end{array}$ & $\begin{array}{l}26.18 \pm .69(\%) \\
24.79 \pm .65 \\
26.23 \pm .69 \\
25.53 \pm .67\end{array}$ \\
\hline $\begin{array}{c}k 1 \text { of ROI } 1 \\
2 \\
3 \\
4\end{array}$ & $\begin{array}{r}.60 \pm 1.19 \\
.88 \pm 1.2 \\
.98 \pm 1.15 \\
2.22 \pm 1.29\end{array}$ & $\begin{array}{l}15.97 \pm .42 \\
16.09 \pm .43 \\
15.40 \pm .41 \\
17.36 \pm .46\end{array}$ & $\begin{array}{l}15.98 \pm .42 \\
16.11 \pm .42 \\
15.43 \pm .41 \\
17.50 \pm .46\end{array}$ \\
\hline $\begin{array}{c}k 1 \text { of ROI } 1 \\
2 \\
3 \\
4\end{array}$ & $\begin{array}{l}1.19 \pm 1.28(\%) \\
2.52 \pm 1.29 \\
1.64 \pm 1.34 \\
.68 \pm 1.13\end{array}$ & $\begin{array}{l}17.19 \pm .45(\%) \\
17.31 \pm .46 \\
18.03 \pm .48 \\
15.22 \pm .4\end{array}$ & $\begin{array}{l}17.23 \pm .45(\%) \\
17.49 \pm .46 \\
18.10 \pm .48 \\
15.23 \pm .4\end{array}$ \\
\hline $\begin{array}{l}x_{1} \text { (coordinate) } \\
x_{2} \text { (coordinate) }\end{array}$ & $\begin{array}{l}.01 \pm .03(\mathrm{~mm}) \\
.02 \pm .03\end{array}$ & $\begin{array}{l}.40 \pm .01(\mathrm{~mm}) \\
.45 \pm .01\end{array}$ & $.40 \pm .01(\mathrm{~mm})$ \\
\hline $\begin{array}{lc} & k 1 \text { of ROI } 1 \\
& 2 \\
\text { Regularization } & 3 \\
(\alpha=10) & 4\end{array}$ & $\begin{array}{r}1.64 \pm 1.26 \\
-.63 \pm 1.19 \\
.54 \pm 1.27 \\
.70 \pm 1.24\end{array}$ & $\begin{array}{l}16.90 \pm .45 \\
15.96 \pm .42 \\
17.07 \pm .45 \\
16.64 \pm .44\end{array}$ & $\begin{array}{l}16.98 \pm .45 \\
15.97 \pm .42 \\
17.08 \pm .45 \\
16.66 \pm .44\end{array}$ \\
\hline
\end{tabular}

Method 1 - Estimation of primary parameters $\boldsymbol{A}$ (ROIs are fixed and are specified from imperfect boundary side information with registration root-mean-square error of $1 \mathrm{~mm}$ ).

Method 2 - Joint estimation of primary parameters $\boldsymbol{A}$ and myocardial boundary parameters $S$ with imperfect boundary side information and perfect registration.

Method 3 (emphasized in this paper) - Joint estimation of primary parameters $A$, boundary parameters $\boldsymbol{S}$, and registration parameters $\boldsymbol{X}$ with imperfect boundary side information.

deviation (SD) of $10 \%$ of their nominal values. If auxiliary boundary measurements ( $\sigma=1 \mathrm{~mm}$ and $\rho=0.3$ ) were available, they were also used as initial estimates. Otherwise, initial boundary parameter estimates were randomized using independent and identically distributed (IID) Gaussian distributions with $1.5 \mathrm{~mm}$ SD. Unless explicitly specified, initial registration parameter estimates were randomized using IID Gaussian distributions with $3 \mathrm{~mm}$ SD.

\section{RESULTS}

\section{A. Boundary Side Information}

Table II lists the statistics of estimates for perfusion $k 1$ and registration parameters $\mathbf{X}$ using the 3 methods. Method 1 , which ignores registration and auxiliary boundary measurement errors, gives significantly higher $k 1$ root-mean-square (RMS) errors and biases compared with other two methods. Method 3, joint estimation of primary, boundary, and registration parameters, provides comparable $k 1$ estimation accuracy in comparison with Method 2, where perfect registration is given. In addition, Method 3 gives registration accuracy of $<0.5 \mathrm{~mm}$. Fig. 2 shows the iteration process using Method 3 (from left to right, top to bottom), which starts with misaligned auxiliary measurements (shifted to the left by $6 \mathrm{~cm}$ ) and ends in well-matched boundary estimates. Notice that the final boundary estimates and the initial estimates are not identical because the final estimates extract information from both the auxiliary and ECT measurements.

\section{B. Regularization}

Table III lists mean RMS errors of $k 1$ (averaged over 4 ROIs) and boundary estimates (averaged over 16 nodes) with different values of regularization parameter $\alpha$. As expected, the mean RMS errors of boundary estimates are sensitive to the selection of $\alpha$. However, the mean RMS errors of $k 1$ estimates are relatively insensitive to the selection of $\alpha$. In addition, results that are not shown here suggest that the sensitivity of boundary estimation to the selection of $\alpha$ decreases as the count rates increases. Fig. 3 shows regularized boundary estimates $(\alpha=10)$ superimposed on filtered-back projection images from the second time interval. The final boundary estimates are smooth and resemble the true elliptical shape.

\section{Side Information versus Regularization}

As seen in Table II, boundary regularization $(\alpha=10)$ gives $k 1$ estimation accuracy comparable with Method 2 as well as Method 3. Mean RMS errors of boundary estimates 
TABLE III

MEAN RMS ERRORS OF $k: 1$ AND BOUNDARY ESTIMATES USING BOUNDARY REGULARIZATION

\begin{tabular}{l|lcl} 
& $k 1$ & endocardial radius & thickness \\
\hline$\alpha=1$ & $16.56(\%)$ & $1.92(\mathrm{~mm})$ & $1.85(\mathrm{~mm})$ \\
$\alpha=10$ & 16.67 & 1.03 & 0.94 \\
$\alpha=100$ & 16.42 & 0.81 & 0.62 \\
$\alpha=1000$ & 15.61 & 1.82 & 2.91
\end{tabular}

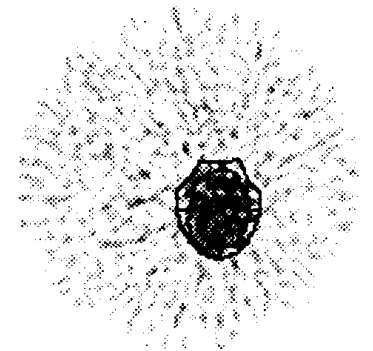

Initial boundary

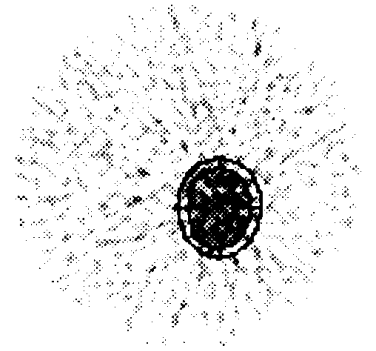

Final boundary
Fig. 3. Regularized boundary estimates ( $\alpha=10$ ) superimposed on filtered-back projection images from the second time interval. The final boundary estimates are smooth and resemble the true eliptical shape.

using regularizers with $\alpha=10$ and $\alpha=100$ (Table III) are comparable with those using Method 3, which gives mean RMS errors of $0.91 \mathrm{~mm}$ for the endocardial radius and 1.01 $\mathrm{mm}$ for the thickness.

\section{DISCUSSION}

In the simulation studies, we have used a simplified object model consisting of homogeneous regions and a stationary heart. Because of the simplification of the model, the simulation results are limited in providing evidence for the practicability of the proposed methods in real data analysis. However, the results are useful for evaluating the potential improvements on the performance of MLMLE following from the use of boundary side information and boundary regularization. In addition, the combination of the simplified model and boundary regularization may already provide a prototype for applying MJMLE to real data analysis.

The simulation results demonstrate that the use of boundary side information stabilizes MJMLE so that it can be applied to gated dynamic studies containing on the order of 0.2 million counts per slice. Furthermore, we show that joint estimation of the registration parameters used to align auxiliary boundary estimates with ECT measurements can be accomplished with accuracy within half a millimeter. This is potentially a very important result since registration of information from other imaging sources has been viewed as a major challenge. It should be emphasized that the case treated here is one in which the physical organ boundary and the functional boundary are identical. The problem becomes more difficult, in the case of infarcted myocardium for instance, when the assumption is not true.
Since boundary side information in the form of MRI or CT measurements is expensive to obtain and is frequently unavailable, it is of interest that boundary regularization is capable of stabilizing the estimation process and gives perfusion and boundary estimates with RMS error equivalent to incorporation of boundary side information with $1 \mathrm{~mm}$ accuracy. The use of boundary regularization requires proper choice of the regularization parameter. Optimization of this parameter is generally computationally expensive to achieve [14]. Nevertheless, a simple analysis such as demonstrated in Table III can be very useful for a given application at given count rates.

As a preliminary trial, we have applied MJMLE with the simplified model and a properly chosen boundary regularizer to analyze PET $N-13$ ammonia data. We used a 3-compartment model to characterize $N-13$ ammonia kinetics. The boundary parameters were initialized from two concentric ellipses approximating the shape of the myocardium. The compartmental parameters ( $k 1, k 2$, and $k 3)$ were initialized according to physiological considerations. The input function was initialized by a nominal shape. Only those projection rays that intersect with the outer ellipse were used in the estimation. When MJMLE was applied without boundary regularization, the estimation process occasionally failed to converge. The incorporation of boundary regularization not only stabilized the estimation process but also significantly reduced the number of iterations needed for convergence. Despite the assumptions of homogeneity and stationarity, the estimated myocardial boundaries agreed well with visual perception of filtered-back projection images. The compartmental parameter estimates were also in the physiologic range. Although the preliminary results are not shown in this paper and will be presented in a future publication, they may serve as additional evidence for the usefulness of boundary regularization.

\section{A. Boundary Alignment}

We have demonstrated that myocardial boundary side information can be accurately aligned through joint estimation of registration parameters with other parameters of interests including myocardial boundary parameters. This alignment method is not limited to myocardial boundaries and can be extended to include both the body contour and other organ boundaries. These auxiliary boundary measurements could share the same registration parameters or they could have different registration parameters. If one ignores the uncertainty in auxiliary boundary measurements, one may simply use a rigid frame for each auxiliary measurement and jointly estimate the registration parameters with other parameters excluding boundary parameters.

Alignment problems related to rotation can be similarly formulated as translation with an additional parameter included in $\mathbf{X}$ in (1) to account for angular misalignment between the two coordinate systems (Fig. 1). We expect this angular registration parameter can be accurately estimated using the method described in this paper, especially when the body contour and other organ auxiliary boundary estimates are included in the model space and, consequently, increase the 
asymmetry of the model. Obviously, to completely solve misalignment problems, it is necessary to extend the alignment method proposed in this paper from 2D to 3D.

It may not be necessary to parameterize the scale differences of ECT/MRI or ECT/CT, which can be easily determined in practice with respect to the standard metric system. One may jointly consider the errors in both scale estimates and boundary estimates. A simple way to do this is to redefine the measurement vector $\tilde{\mathbf{R}}$ to include the scale measurement in a combined measurement vector $\tilde{\mathbf{T}}$ as

$$
\tilde{\mathbf{T}}=\tilde{c} \cdot \tilde{\mathbf{R}}
$$

Depending on the distribution of $\tilde{c}$, one may be able to analytically derive the distribution of $\tilde{\mathbf{T}}$ from both $\tilde{c}$ and $\tilde{\mathbf{R}}$.

\section{APPENDIX A}

To appropriately model the errors in auxiliary boundary measurements, we consider a Markov-type formulation for the covariance matrix $\mathbf{V}_{\tilde{\mathbf{R}}}$, i.e. we assume the errors in node radius measurements are correlated in a predictable fashion. We write

$$
\mathbf{V}_{\tilde{\mathbf{R}}}=\left[\begin{array}{cc}
\mathbf{V}_{a} & 0 \\
0 & \mathbf{V}_{b}
\end{array}\right]
$$

where by assuming endocardial node radii and epicardial node radii are uncorrelated with each other, the errors in the two sets of auxiliary measurements are individually specified in the subcovariance matrices $V_{a}$ and $V_{b}$. We assume $V_{a}$ and $V_{b}$ have the following structure

$$
\mathbf{V}_{a(\text { or } h)}=\sigma^{2} \cdot\left[\begin{array}{cccccccc}
\rho^{0} & \rho^{1} & \cdot & \cdot & \rho^{\frac{n}{2}} & \rho^{\frac{n}{2}-1} & \cdot & \rho^{1} \\
\rho^{1} & \rho^{0} & \rho^{1} & & & \cdot & & \\
\cdot & \rho^{1} & \rho^{0} & \cdot & & & \cdot & \\
\cdot & & \cdot & \cdot & \cdot & & & \rho^{\frac{n}{2}} \\
\rho^{\frac{n}{2}} & & & \cdot & \cdot & \cdot & & \\
\rho^{\frac{n}{2}-1} & \cdot & & & \cdot & & & \\
\cdot & & \cdot & & & & & \rho^{1} \\
\rho^{1} & \rho^{2} & & \rho^{\frac{n}{2}} & & & \rho^{1} & \rho^{0}
\end{array}\right]
$$

where $\sigma$ is a scalar characterizing the magnitude of the errors, $n$ is an even number specifying the number of nodes, and $\rho(0<\rho<1)$ with varying powers describes distancedependent correlations among radius measurements. This structure reflects the fact that nearby hand-drawn nodes will have more correlated errors than distant nodes.

\section{APPENDIX B}

We regularize the epicardial radii and angular thicknesses using the following regularizer

$$
\begin{aligned}
\Phi(\mathbf{R})= & \left\{\left(r_{b}(n)-r_{a}(n)\right)-\left(r_{b}(1)-r_{a}(1)\right)\right\}^{2} \\
& +\sum_{i=1}^{n=1}\left\{\left(r_{b}(i)-r_{a}(i)\right)-\left(r_{b}(i+1)-r_{a}(i+1)\right)\right\}^{2} \\
& +\left(r_{b}(n)-r_{b}(1)\right)^{2}+\sum_{i=1}^{n-1}\left(r_{b}(i)-r_{b}(i+1)\right)^{2}(\mathrm{~B} .1)
\end{aligned}
$$

where $n$ is the number of the nodes, and $r_{a}(i)$ and $r_{b}(i)$ are the $i$-th endocardial and epicardial node radii. Let $\mathbf{R}_{a}$ and $\mathbf{R}_{b}$ be the vector concatenations of endocardial and epicardial node radii. We define

$$
\Delta \mathbf{R}=\left[\begin{array}{c}
\Delta \mathbf{R}_{a} \\
\Delta \mathbf{R}_{b}
\end{array}\right]=\left[\begin{array}{c}
r_{a}(1)-r_{a}(2) \\
r_{a}(2)-r_{a}(3) \\
\cdot \\
r_{a}(n)-r_{a}(1) \\
r_{b}(1)-r_{b}(2) \\
r_{b}(2)-r_{b}(3) \\
\cdot \\
r_{b}(n)-r_{b}(1)
\end{array}\right]
$$

We have' shown [18] that (B.1) is equivalent to $\Phi(\mathbf{R})=$ $\Delta \mathbf{R}^{\mathbf{T}} \cdot \mathbf{W} \cdot \boldsymbol{\Delta} \mathbf{R}$ with $\mathbf{W}$ specified by

$$
\mathbf{W}=\left[\begin{array}{rr}
\mathbf{I} & -\mathbf{I} \\
-\mathbf{I} & 2 \mathbf{I}
\end{array}\right]
$$

In the following derivations of the Hessian of $\Phi(\mathbf{R})$ with respect to boundary parameters $\mathbf{S}$, we use the following relation

$$
\mathbf{S}=\mathbf{G} \cdot \mathbf{R}=\left[\begin{array}{rr}
\mathbf{I} & \mathbf{0} \\
-\mathbf{I} & \mathbf{I}
\end{array}\right] \cdot\left[\begin{array}{l}
\mathbf{R}_{a} \\
\mathbf{R}_{b}
\end{array}\right]=\left[\begin{array}{ccc} 
& \mathbf{R}_{a} & \\
\mathbf{R}_{b} & - & \mathbf{R}_{a}
\end{array}\right] .
$$

Since $\Phi(\mathbf{R})$ is quadratic, the Hessian $\nabla_{\mathbf{S}}^{2} \Phi(\mathbf{R})$ is given by

$$
\begin{aligned}
& \nabla_{\mathrm{S}}^{2} \Phi(\mathbf{R}) \\
& =\mathbf{G}^{-1} \cdot \nabla_{\mathbf{R}}^{2} \Phi(\mathbf{R}) \cdot\left[{ }^{-1}{ }^{-1}\right]^{T} \\
& =2 \boldsymbol{c}^{-1} \cdot\left[\begin{array}{cc}
\nabla_{\mathbf{R}_{a}} \Delta \mathbf{R}_{a} & \mathbf{0} \\
\mathbf{0} & \nabla_{\mathbf{R}_{b}} \Delta \mathbf{R}_{b}
\end{array}\right] \cdot \mathbf{W} \\
& \cdot\left[\begin{array}{cc}
\nabla_{\mathbf{R}_{a}} \Delta \mathbf{R}_{a} & \mathbf{0} \\
\mathbf{0} & \nabla_{\mathbf{R}_{b}} \Delta \mathbf{R}_{b}
\end{array}\right]^{T} \cdot\left[\mathbf{G}^{-1}\right]^{T} \\
& =2 \mathbf{G}^{-1} \\
& \cdot\left[\begin{array}{cc}
\nabla_{\mathbf{R}_{a}} \Delta \mathbf{R}_{a} \cdot\left[\nabla_{\mathbf{R}_{a}} \Delta \mathbf{R}_{a}\right]^{T} & -\nabla_{\mathbf{R}_{a}} \Delta \mathbf{R}_{a} \cdot\left[\nabla_{\mathbf{R}_{b}} \Delta \mathbf{R}_{b}\right]^{T} \\
-\nabla_{\mathbf{R}_{b}} \Delta \mathbf{R}_{b} \cdot\left[\nabla_{\mathbf{R}_{a}} \Delta \mathbf{R}_{a l}\right]^{T} & 2 \nabla_{\mathbf{R}_{\mathbf{b}}} \Delta \mathbf{R}_{b} \cdot\left[\nabla_{\mathbf{R}_{b}} \Delta \mathbf{R}_{b}\right]^{T}
\end{array}\right] \\
& \cdot\left[\mathbf{G}^{-1}\right]^{T} \text {. }
\end{aligned}
$$

Since the gradients of either $\Delta \mathbf{R}_{a}$ or $\Delta \mathbf{R}_{b}$ with respect to either $\mathbf{R}_{a}$ or $\mathbf{R}_{b}$ are identical, we define the product of any pair of them as $\mathbf{Q}$. It can be easily shown that

$$
\mathbf{Q}=\left[\begin{array}{cccccc}
2 & -1 & & & & -1 \\
-1 & 2 & \cdot & & & \\
& \cdot & \cdot & \cdot & & \\
& & \cdot & \cdot & & \\
& & & & & -1 \\
-1 & & & & -1 & 2
\end{array}\right]
$$

Thus, (B.5) can be simplified as

$$
\nabla_{\mathbf{S}}^{2} \Phi(\mathbf{R})=\mathbf{2}\left[\begin{array}{cc}
\mathbf{Q} & \mathbf{Q} \\
\mathbf{Q} & \mathbf{2 Q}
\end{array}\right]
$$




\section{REFERENCES}

[1] P. Chiao, W. L. Rogers, N. H. Clinthorne, A. Fessler, and A. O. Hero, "Model-based estimation for dynamic cardiac studies using ECT," IEEE Trans. Med. Imag., vol. 13, no. 2, pp. 217-226, 1994.

[2] E. J. Hoffman, M. E. Phelps, G. Wisenberg, H. R. Schelbert, and D. E. Kuhl, "Electrocardiographic gating in positron emission computed tomography," J. Comput. Assist. Tomogr., vol. 3, pp. 733-9, 1979.

[3] C. A. Pelizzari, G. T. Chen, D. R. Spelbring, R. R. Weichselbaum, and C. T. Chen, "Accurate three-dimensional registration of CT, PET and/or MR images of the brain," J. Compur. Assist. Tomogr., vol. 13, pp. 20-6, 1989.

[4] C. C. Meltzer, R. N. Bryan, H. H. Holcomb, A. W. Kimball, H S. Mayberg, B. Sadzot, J. P. Leal, H. J. Wagner, and J. J. Frost, "Anatomical localization for PET using MR imaging," J.Comput. Assist. Tomogr., vol. 14, pp. 418-26, 1990.

[5] A. C. Evans, C. Beil, S. Marrett, C. J. Thompson, and A. Hakim, "Anatomical-functional correlation using an adjustable MRI-based region of interest atlas with positron emission tomography," J. Cereb. Blood. Flow. Metab., vol. 8, pp. 513-30, 1988.

[6] R. Dann, J. Hoford, S. Kovacic, M. Reivich, and R. Bajcsy, "Evaluation of elastic matching system for anatomic (CT, MR) and functional (PET) cerebral images," J. Comput. Assist. Tomogr., vol. 13, pp. 603-11, 1989

[7] C. T. Chen, X. Ouyang, W. H. Wong, V. E. Johnson, C. Ordonez, and C. E. Metz, "Sensor fusion in image reconstruction," IEEE Trans. NuCl. Sci., vol. NS-38, pp. 687-692, 1991.

[8] V. E. Johnson, W. H. Wong, X. Hu, and C. T. Chen, "Image restoration using gibbs priors: boundary modeling, treatment of blurring and selection of hyperparameter," IEEE Trans. Pattern, Anal. Mach. Intell., vol. 13 , pp. $413-425,1991$.
[9] V. Torre and T. A. Poggio, "On edge detection," IEEE Trans. Pattern Anal. Mach. Intell., vol. 8, pp. 147-163, 1986.

[10] D. Terzopoulos, "Regularization of inverse visual problems involving discountinuities," IEEE Trans. Pattern Anal. Mach. Intell., vol. PAMI-8, pp. 413-424, 1986.

[11] N. B. Karayiannis and A. N. Venetsanopoulos, "Regularization theory in image restoration-the stabilizing functional approach," IEEE Trans. Accoust., Speech, and Signal Processing, vol. 38, pp. 1155-1179, 1990.

[12] S. Kawata and O. Nalcioglu, "Constrained iterative reconstruction by the conjugate gradient method," IEEE Trans. Med. Imag., vol. MI-4, pp. $65-71,1985$.

[13] A. N. Tikhonov and V. Y. Arsenin, Solutions of III-Posed Problems. Washington, DC: Winston, 1977.

[14] G. H. Golub, M. Heath, and G. Wahba, "Generalized cross-validation as a method for choosing a good ridge parameter," Technometrics, vol. 21, pp. 215-223, 1979.

[15] A. M. Thompson, J. C. Brown, J. W. Kay, and D. M. Titterington, "A study of methods of choosing the smoothing parameter in image restoration by regularization," IEEE Trans. Pattern Anal. Mach. Intell., vol. pp. 326-339, 1991.

[16] R. E. Carson, "A maximum likelihood method for region-of-interest evaluation in emission tomography," J. Comput. Assist. Tomogr., vol. 10, pp. 654-63, 1986.

[17] R. E. Carson and K. Lange, "The EM parametric image reconstruction algorithm," J. Amer. Statist. Assoc., vol. 80, pp. 20-2, 1985.

[18] P. Chiao, "Parameter estimation strategies for dynamic cardiac studies using emission computed tomography," Ph.D. dissertation, University of Michigan, 1991.

[19] R. H. Huesman and B. M. Mazoyer, "Kinetic data analysis with a noisy input function," Phys. Med. Biol., vol. 32, pp. 1569-79, 1987. 